\title{
Updating objects in visual short-term memory is feature selective
}

\author{
Philip C. Ko And Adriane E. Seiffert \\ Vanderbilt University, Nashville, Tennessee
}

\begin{abstract}
The purpose of this study was to examine whether the process of updating information in visual short-term memory (VSTM) is object based. We investigated whether modifying the memory of one feature of an object would automatically promote refreshing the memory of all of its other features. The results showed that the facilitative effect of updating was specific to the updated feature of an object and did not spread to its nonupdated features. This feature-selective effect suggests that updating VSTM is not object based (Experiment 1), even though storage was object based (Experiment 2). Control experiments ruled out strategy-based (Experiment 3) and stimulus-related (Experiments 4-6) accounts. Feature-selective updating may indicate that the mechanism used to modify the contents of memory may have a different basis than that used to encode or store information in memory.
\end{abstract}

Updating refers to the modification of short-term memory by adding new information and/or replacing old information. This is an important ability in daily life-for example, when creating a mental list and revising it as new priorities arise. Imagine you are at the grocery store and you get a call telling you to buy milk, but not butter. You can modify your working memory to incorporate this new information and end up with a new mental list. Updating is an executive function and is not constrained by the limits of short-term memory capacity (Morris \& Jones, 1990). Some researchers suggest that updating is functionally distinct from other executive functions, such as inhibition and task switching (Miyake, Friedman, Emerson, Witzki, \& Howerter, 2000), and is the only one of these functions related to intelligence measures (Friedman et al., 2005). Here, we will use the term updating to refer to any modification to the contents of working memory that necessitates combining new information with stored information, including modifying the memory of the attributes of an object. Updating tasks have been useful in examining other cognitive processes (Garavan, 1998; Oberauer, 2002), but the updating process itself has been examined in only a few studies (Bao, Li, \& Zhang, 2007; Kessler \& Meiran, 2006; Morris $\&$ Jones, 1990). The purpose of this investigation was to examine properties of updating; specifically, we examined whether updating visual short-term memory (VSTM) is an object-based process.

Luck and Vogel (1997) provided the first evidence suggesting that object representations were the units of storage in VSTM. As has been done in many experiments of VSTM, participants' memories were tested with a delayed change-detection task. The participants studied a brief sample array of a variable number of colored and tilted bars. After a blank delay period, another array of objects was shown that either was identical to the sample or differed by one color or orientation feature. Recognition performances indicated that the participants' VSTM capacity was limited to about four items. More important, this capacity was the same whether people were remembering a single feature (e.g., a color) or a conjunction of multiple features (e.g., a color and an orientation). Because more information about the objects could be remembered without cost, this result indicated that information in VSTM could be represented at the level of unified objects (see also Vogel, Woodman, \& Luck, 2001). Although the idea of object-based capacity is debated (Olson \& Jiang, 2002; Wheeler \& Treisman, 2002), the bulk of the research supports the main contention that object-based representations are used in VSTM.

Following these findings of object-based storage in VSTM, the purpose of the present study was to examine whether another use of VSTM - specifically, updating was also object based. We asked whether updating memory would lead to reprocessing of all the features of a target object. In other words, does updating one feature of an object in memory automatically refresh all other features of the same object? As an example, imagine that you get a call telling you that the milk you should purchase has a red label, not a blue one. While you are updating your memory of the color of the label, do you also simultaneously refresh your memory for the shape of the container? Although one can imagine that a person could use verbal cues in this situation, VSTM may also be used. If VSTM stores information as object representations, one might expect that any process, such as updating, that 
affected one feature of the object's representation would also have an effect on the other features. Some previous research has already demonstrated a tendency for people to refresh existing information in working memory when updating part of working memory (Kessler \& Meiran, 2006). Here, we have explored how updating works on visual information by testing whether updating is object based - in other words, whether updating necessarily invokes refreshing of all visual features of an object in memory. In most of the experiments below, we investigated VSTM, but our final experiment suggested that our findings generalize to verbal short-term memory.

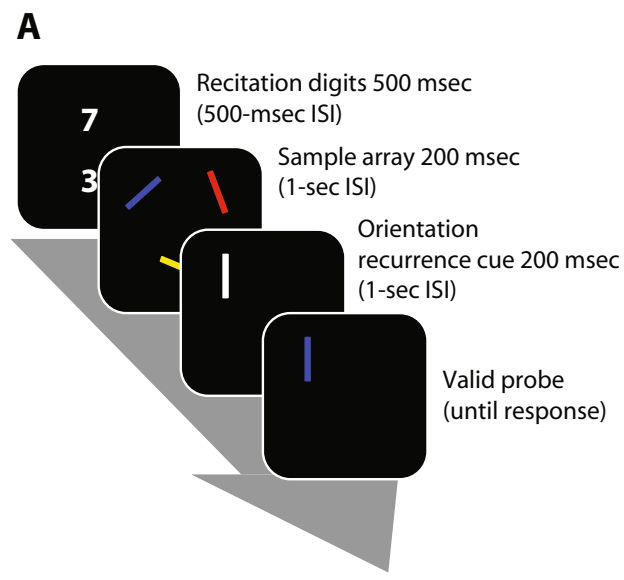

B

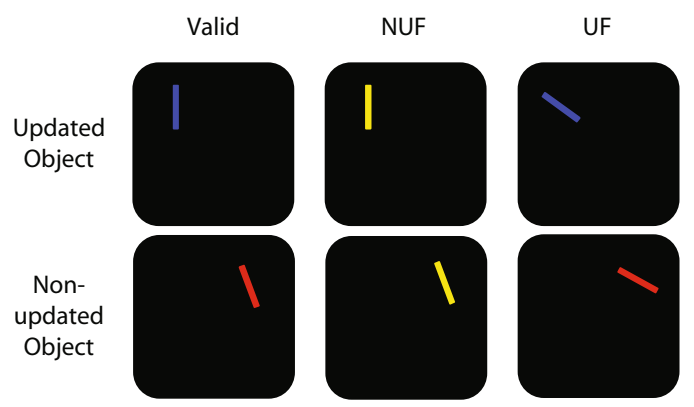

Figure 1. A schematic of the general methodology. (A) The trial starts with the two digits for the articulatory suppression task. After a 500-msec blank interstimulus interval (ISI), the sample array of three colored bars was shown for 200 msec. After a 1-sec blank ISI, the recurrence cue-here, a white vertical bar-was shown in place of the blue, $45^{\circ}$ tilted bar in the sample. For this example, the participants would have been instructed to update their memory by changing the orientation of the blue tilted bar to be a blue vertical bar. The probe that appeared at the end of the trial was valid if it matched the updated information, as it does in this example. (B) Examples of probes that could follow the trial depicted in Panel A. Types of probes for the updated objects are shown in the top row and for the nonupdated objects in the bottom row. Valid probes of updated objects were the combination of features from the sample and the recurrence cue (e.g., a blue vertical bar). Invalid probes either changed the nonupdated feature of the object (NUF), which was color in this example, or the updated feature (UF), which was orientation here.

\section{EXPERIMENT 1}

The purpose of Experiment 1 was to examine whether updating proceeded in an object based manner. We utilized a change-detection task, typically used to assess VSTM, with an additional procedural step (see Figure 1A). Participants viewed a sample array of multiple colored bars, followed by a delay and a probe display consisting of a single object. In addition, between the sample and the probe, another stimulus appeared at one of the object locations to cue the participants to modify their memory of the corresponding object. We called this stimulus a recurrence cue, which refers to the idea that the new stimulus is to be considered part of the corresponding object, if it was to appear again. If the recurrence cue was a colored dot, the participants were to modify their memory of the object that had previously appeared in the same location as the recurrence cue so that its color matched that of the recurrence cue. If the cue was a white bar, they were to modify the orientation of the object to match that of the recurrence cue. If memory updating is object based, the updating process should affect all features of the object indicated by the recurrence cue. To test the memory for both features of the objects, changes were applied to a feature dimension that was either the same as or different from the recurrence cue. For example, if the recurrence cue indicated updating the color of the object, either the color of the probed object could be changed or the orientation of the probed object could be changed. These trials were referred to as the updated feature (UF) or nonupdated feature (NUF) trials, respectively (Figure 1B).

Experiment 1 was designed to examine three hypotheses, all of which shared the following assumption. It was assumed that memory for information indicated by the recurrence cue would be facilitated. Recent studies have shown that shifts of attention to items in memory result in an enhanced representation or prioritization of the item (Griffin \& Nobre, 2003; Landman, Spekreijse, \& Lamme, 2003; Makovski \& Jiang, 2007; Makovski, Sussman, \& Jiang, 2008). The object-based hypothesis proposes that the effect of updating one feature of an object should spread to all of its features. This predicts performance benefits for both the updated feature and the nonupdated feature in the updated object (Figure 2A). This result could come about if, when updating the memory of one feature, the memory of the other feature of the object is refreshed, so that both features share facilitation of memory performance. The feature-based hypothesis proposes that the effect of updating one feature results in sensitivity to all values of that feature dimension in memory. This hypothesis predicts superior change detection for the updated feature, regardless of whether an updated or nonupdated object was probed (Figure 2B). Finally, the feature-selective hypothesis proposes that the facilitative effect of updating will be restricted to only the updated feature and object (Figure 2C). This hypothesis contrasts with that of the object-based hypothesis in that it predicts that the effect of updating will not spread to all features of the updated object. It contrasts with that of the featurebased hypothesis in that it predicts that the effect of updating will not spread to all objects. 


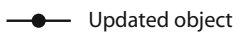

- o - Nonupdated object

A

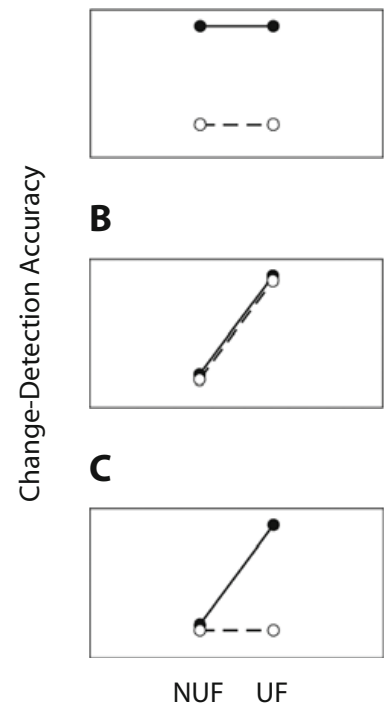

Figure 2. Predictions made by (A) the object-based hypothesis, (B) the feature-based hypothesis, and (C) the feature-selective hypothesis. NUF, nonupdated feature; UF, updated feature.

\section{Method}

Participants. The participants were 11 adult volunteers ( $7 \mathrm{fe}-$ male) with a mean age of 25.45 years $(S D=4.82)$, recruited from the Nashville community through the Psychology Research Sign-up System at Vanderbilt University. They all participated in exchange for $\$ 10$ per hour.

Apparatus and Stimuli. The stimuli were presented in MATLAB using the Psychophysics Toolbox extension (Brainerd, 1997; Pelli, 1997) on an eMac G4 with a CRT monitor at a resolution of $1,024 \times$ 768 pixel and a refresh rate of $89 \mathrm{~Hz}$. The participants used a headrest, which positioned them at approximately $57 \mathrm{~cm}$ from the monitor. Vocal utterances were recorded with an InSync gooseneck microphone connected with a Griffin iMic USB converter.

The stimuli were colored bars subtending $0.5^{\circ} \times 2^{\circ}$ visual angle. These objects were positioned on three equidistant points of an imaginary circle with a diameter of $8.5^{\circ}$ visual angle, such that the overall spatial configuration resembled an inverted triangle. To create a set of 12 colors that were readily discriminable, we selected 12 equidistant points of a circle centered on the white spot in $1976 \mathrm{CIE}$ $L^{*} u^{*} v^{*}$ color space (CIE, 1986; Schanda, 2007) and with a radius that maximized saturation to the limits of the monitor. In separate visual search experiments, we determined that second neighbors of this color set are easily discriminable from each other, because search slopes were flat (data not shown). Only second-neighbor colors appeared in a given trial of this experiment. Orientations were eight angles increasing from $0^{\circ}$ (vertical) in $22.5^{\circ}$ increments to $157.5^{\circ}$, and only second-neighbor orientations appeared in a given trial (e.g., $0^{\circ}, 45^{\circ}$, and $90^{\circ}$ ).

Procedure. At the beginning of every trial, two vertically aligned digits appeared in the center of the screen for $500 \mathrm{msec}$, followed by a blank $500-\mathrm{msec}$ interstimulus interval (ISI). The participants recited these numbers aloud into the microphone for the duration of the trial. Concurrent performance of this articulatory suppression task likely reduced verbal encoding strategies. Next, the sample array appeared for $200 \mathrm{msec}$, followed by a blank 1-sec ISI. Objects in the sample array were three colored bars, each with unique colors and orientations (see the Apparatus and Stimuli section). Then, the recurrence cue appeared for $200 \mathrm{msec}$ in the same location as a randomly selected object in the sample array, followed by a blank 1 -sec ISI. On half of the trials, the recurrence cue was a white bar at an orientation that was not shown in the sample array. On the other trials, the recurrence cue was a dot (subtending $0.7^{\circ}$ in diameter) in a color that was not shown in the sample array. The participants were instructed to update their memory of the orientation or color of the object in the corresponding location (Figure 1A).

A single bar was randomly selected to appear in the probe display. On half of the trials, the probe was valid. Valid probes of updated objects appeared with the updated feature modified to match that of the recurrence cue (Figure 1A). Valid probes of nonupdated objects appeared as they did in the sample display (Figure 1B, bottom row, left). On the other half of the trials, the probe was invalid, with the change applied in one of two ways. In NUF trials, the change was applied to the feature dimension different from that of the recurrence cue. In UF trials, the change was applied to the same feature dimension as that of the recurrence cue. Changes applied on invalid trials were features from noncorresponding objects (i.e., a feature swap). The invalid probes of the updated objects were never the same as the objects in the sample array. To prevent correlation between which object was updated and which object was probed for memory, the selection of the object to be updated was made randomly, such that each object had equal probability of being probed. This resulted in an uneven number of trials in which updated or nonupdated objects were probed, but the variation in the number of trials for each condition remained relatively low across participants. From a total of 480 trials, nonupdated objects were probed in a mean of 318.9 trials $(S D=6.59)$ and updated objects were probed in a mean of 161.09 trials $(S D=6.59)$.

\section{Results and Discussion}

The alpha level was initially set to .05 for all omnibus tests in this study. The .05 alpha level was Bonferroni corrected for multiple paired comparisons. The measures of effect size reported are $\eta_{\mathrm{p}}^{2}$ and Cohen's $d$. Data for the articulatory suppression task were scored as the mean number of articulations per trial. The articulation data were separated according to whether updated or nonupdated objects were probed and then submitted to a $2 \times 3$ within-subjects ANOVA to examine the effects of object (nonupdated, updated) and probe (valid, NUF, UF). The results showed no main effects, and the interaction was not significant (all $F \mathrm{~s}<1$ ), showing no differences in the rate of articulations across conditions. This suggested that there were no changes in verbal encoding that could have explained the performances in the visual task. The mean number of articulations per trial was $4.62(S D=0.13)$.

Change-detection data were scored as the proportion accurate across trial repetitions. The accuracy data were submitted to a $2 \times 3$ within-subjects ANOVA to examine the effects of object (nonupdated, updated) and probe (valid, NUF, UF). The results showed main effects of object $\left[F(1,10)=29.7, p<.001, \eta_{\mathrm{p}}^{2}=.75\right]$ and probe $\left[F(2,20)=6.04, p<.01, \eta_{\mathrm{p}}^{2}=.37\right]$ and a significant object $\times$ probe interaction $\left[F(2,20)=5.43, p<.05, \eta_{\mathrm{p}}^{2}=\right.$ .35]. This significant interaction fit the prediction from the feature-selective hypothesis but did not fit the other hypotheses. Paired comparisons showed that, in valid trials, there was no significant difference in change-detection accuracy between updated objects $(80 \%)$ and nonupdated objects $(71 \%)[t(10)=1.78, p=.1, d=0.79]$. Since our 
a priori hypotheses only made predictions based on the invalid trials (NUF and UF), the remaining analyses focused on these trials. In NUF trials, there was no significant difference between updated objects $(57 \%)$ and nonupdated objects $(59 \%)[t(10)=0.91, p=.38, d=0.12]$. In UF trials, accuracy for updated objects $(81 \%)$ was superior to that for nonupdated objects $(58 \%)[t(10)=3.9, p<.01$, $d=1.69]$. In updated objects, there was superior accuracy in the UF trials than in the NUF trials $[t(10)=3.23, p<$ $.01, d=1.39]$ but in nonupdated objects, there was no difference in the same contrast $[t(10)=0.52, p=.61$, $d=0.06]$ (see Figure 3). To examine whether these results were selectively determined by either color or orientation updates, we separated the trials according to which feature was updated and submitted the data to an object (nonupdated, updated) $\times$ feature (color, orientation) $\times$ probe (valid, NUF, UF) ANOVA. This analysis revealed main effects of object $\left[F(1,10)=29.7, p<.001, \eta_{\mathrm{p}}^{2}=.75\right]$, feature $\left[F(1,10)=7.77, p<.05, \eta_{\mathrm{p}}^{2}=.44\right]$, and probe $\left[F(2,20)=6.04, p<.01, \eta_{\mathrm{p}}^{2}=.38\right]$. The main effect of feature showed higher performances with color updates $(\bar{X}=0.7, S E=0.022)$ than with orientation updates $(\bar{X}=$ $0.66, S E=0.026)$. The object $\times$ feature interaction was not significant $\left[F(1,10)=1.58, p=.23, \eta_{\mathrm{p}}^{2}=.13\right]$, nor was the feature $\times$ probe interaction $[F(2,20)=2.8, p=$ $\left..08, \eta_{\mathrm{p}}^{2}=.22\right]$. The primary effect of our interest - the object $\times$ probe interaction-was significant $[F(2,20)=$ 5.43, $\left.p<.05, \eta_{\mathrm{p}}^{2}=.35\right]$, but more important, the threeway interaction was not significant $[F(2,20)=2.57$, $\left.p=.1, \eta_{\mathrm{p}}^{2}=.2\right]$, indicating that color and orientation updates did not have a differential effect on the object $X$ probe interaction.

These results clearly support the feature-selective hypothesis (Figure 2C) and reject the feature-based and object-based hypotheses. Memory for the updated feature was facilitated, but only in the updated object; this result rejects the feature-based hypothesis. Memory for the updated feature of the updated object was superior to that of its nonupdated feature, indicating that the facilitative

\section{Experiment 1: Conjunction Updating}

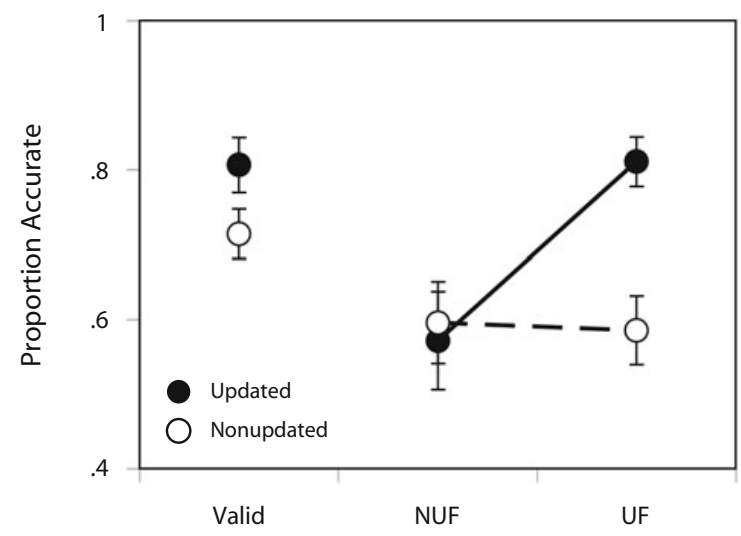

Figure 3. Accuracy data for Experiment 1. The error bars represent $1 S E M$. NUF, nonupdated feature; UF, updated feature. effect of updating was found only for the updated feature; this result rejects the object-based hypothesis. Crucially, memory for the nonupdated feature of the updated object was no better than memory for the nonupdated objects. This result suggests that the facilitative effects of updating do not spread to the nonupdated features of the object. However, there are at least two alternative accounts of this data. First, it is possible that the participants did not memorize the visual stimuli as objects. Information may not have been stored in an object-based manner, which we address in Experiment 2. Alternatively, the participants may have strategically encoded the information as separate features in order to perform the updating task, which we address in Experiment 3. Second, the nature of the recurrence cue may have prevented the participants from forming an updated object (Experiments 4 and 5).

\section{EXPERIMENT 2}

One reason that Experiment 1 may have shown featureselective updating rather than an object-based effect was that information was not stored as objects in VSTM. There is evidence that, in some circumstances, object-based storage does not occur (Olson \& Jiang, 2002; Wheeler \& Treisman, 2002). The purpose of Experiment 2 was to examine whether the object-based effect of storage could be replicated with our stimuli and environment. Object-based storage is reflected by similar capacities for simple features and more complex conjunctions of features (Luck \& Vogel, 1997). In this experiment, the participants engaged in two tasks: the updating task (modified from Experiment 1) and a standard maintenance task. In the maintenance task, we evaluated object-based storage by manipulating the stimulus type and set size (modified from Luck \& Vogel, 1997). The participants performed the tasks in separate blocks, and task order was counterbalanced. This allowed us to observe whether visual memory was objectbased at the same time as replicating Experiment 1 with slightly different stimuli.

\section{Method}

Participants. The participants were 11 Vanderbilt University undergraduate students (6 females), with a mean age of 18.72 years $(S D=1.01)$. All of the participants were recruited through the Psychology Research Sign-up System at Vanderbilt University, and participated in exchange for credits toward the requirements of undergraduate psychology classes.

Apparatus and Stimuli. The apparatus was identical to that of Experiment 1. The stimuli were adapted to better match those of Luck and Vogel (1997). The sample array objects used in the maintenance task were colored squares, white oriented bars, or colored oriented bars against a black background. Colored oriented bars were used for the sample array in the updating task. The squares subtended $0.7^{\circ} \times 0.7^{\circ}$ visual angle, and the bars subtended $1.2^{\circ} \times$ $0.2^{\circ}$. The objects were centered on the points of a $5 \times 5$ grid that spanned $10^{\circ} \times 7^{\circ}$. The points on the grid were equally spaced by $2.5^{\circ}$ in the horizontal plane and $1.75^{\circ}$ on the vertical plane. Positions were randomly jittered, such that an object could be displaced by up to $\pm 0.25^{\circ}$ in the horizontal plane and $\pm 0.175^{\circ}$ in the vertical plane. The colors and orientations were identical to those used in Experiment 1.

Procedure. The maintenance task proceeded as follows. Two vertically aligned digits appeared in the center of the screen for $500 \mathrm{msec}$, 
followed by a blank 500-msec ISI. Then, a sample array composed of $2,4,6$, or 8 objects appeared for $100 \mathrm{msec}$, followed by a 1 -sec blank ISI. The objects were colored squares, white oriented bars, or conjunctions of color and orientation. The objects could share the same color and/or orientation, but location was unique to each object (i.e., Vogel et al., 2001). Then another array of objects appeared until the participants provided a response. On half of the trials, this test array was identical to the sample array, and on the other half of the trials, it differed by one object. On different trials, a randomly chosen object appeared with a different feature value than that of the corresponding object in the sample array. The different feature value was directly opposite of the sample feature value in either color or orientation space; for example, a yellow square would be replaced by a blue one, and a vertically oriented bar would be replaced by a horizontal one. In the conjunction condition, there were an equal number of color and orientation changes to the changed object. The updating task was identical to that in Experiment 1, except that the stimuli were the same as those used in the maintenance task. The tasks were blocked in the session, and task order was counterbalanced across participants.

\section{Results and Discussion}

The results from the maintenance task replicated previous work showing object-based storage in VSTM (Luck \& Vogel, 1997). Following Luck and Vogel, the data for the maintenance task (Figure 4A) were scored as capacity estimates $(K)^{1}$ according to Cowan's (2001) formula in order to understand the results as they related to the number of objects in the display:

$$
\begin{aligned}
K= & (\text { hits }+ \text { correct rejections }-1) \\
& \times \text { the number of objects in the display }
\end{aligned}
$$

These data were submitted to a $3 \times 4$ within-subjects ANOVA to examine the effects of stimulus (color, orientation, conjunction) and set size $(2,4,6,8)$. The results revealed main effects of stimulus $[F(2,20)=30.29, p<$ $\left..001, \eta_{\mathrm{p}}^{2}=.75\right]$ and set size $\left[F(3,30)=5.58, p<.01, \eta_{\mathrm{p}}^{2}=\right.$ $.36]$, as well as a significant stimulus $\times$ set size interaction $\left[F(6,60)=4.3, p<.01, \eta_{\mathrm{p}}^{2}=.3\right]$.

These effects were driven by differences between the orientation trials and the other stimulus types. To confirm this, a separate ANOVA, which excluded the orientation data, was conducted to examine the effects of stimulus (color, conjunction) and set size $(2,4,6,8)$. This showed no effect of stimulus $\left(F<1, p=.98, \eta_{\mathrm{p}}^{2}<.01\right)$ but did show a main effect of set size $[F(3,30)=3.19, p<.05$, $\left.\eta_{\mathrm{p}}^{2}=.24\right]$. The interaction was not significant $(F<1$, $\left.p=.45, \eta_{\mathrm{p}}^{2}<.1\right)$. These results showed that the capacity for conjunctions matched that for the most difficult feature, which was color (Figure 4A), indicating object-based storage. This replicated previous findings supporting the conclusion that VSTM has an object-based capacity (Luck \& Vogel, 1997; Vogel et al., 2001).

The results from the updating task replicated the feature-selective effect from Experiment 1 . The data for the updating task (Figure 4B) were scored as proportion accurate. The accuracy data were submitted to a $2 \times 3$ ANOVA to examine the effects of object (nonupdated, updated) and probe (valid, NUF, UF). The results revealed a nonsignificant effect of object $[F(1,10)=4.29, p=.06$, $\left.\eta_{\mathrm{p}}^{2}=.3\right]$, a significant effect of probe $[F(2,20)=6.65$, $\left.p<.01, \eta_{\mathrm{p}}^{2}=.4\right]$, and a significant object $\times$ probe interaction $\left[F(2,20)=30.13, p<.001, \eta_{\mathrm{p}}^{2}=.75\right]$. Paired

\section{A Experiment 2: VSTM Capacity}

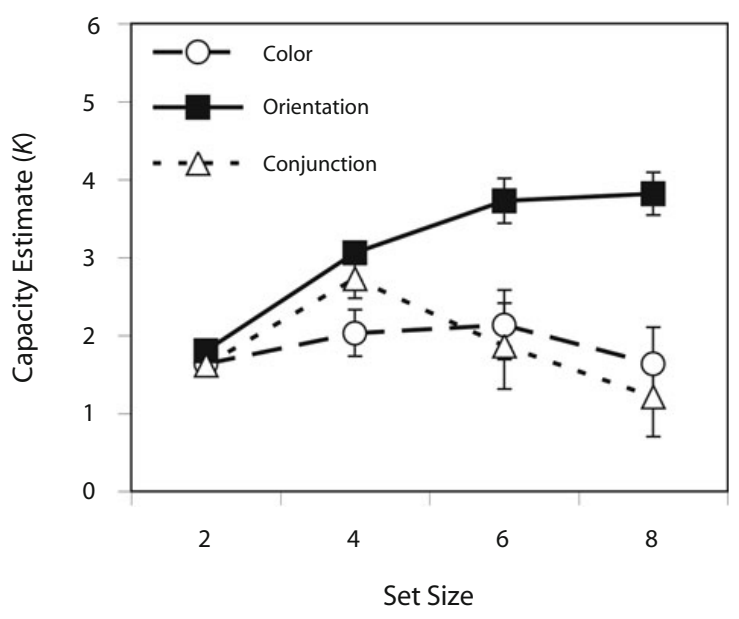

B Experiment 2: Conjunction Updating

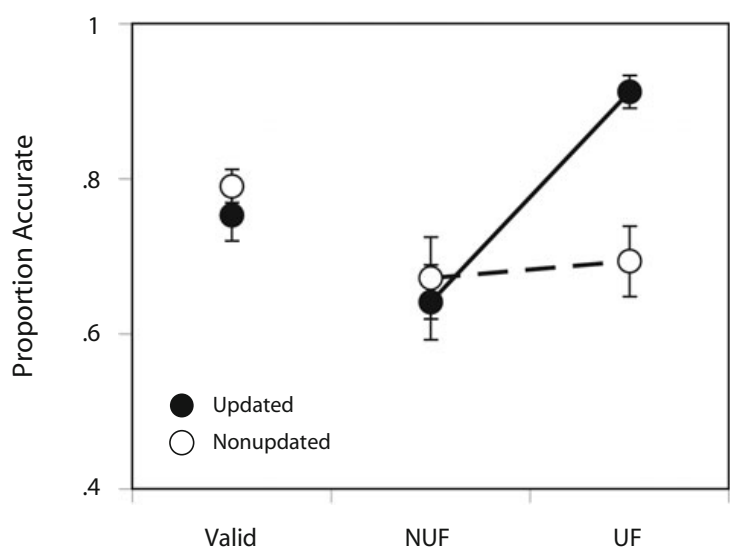

Figure 4. Results from Experiment 2. (A) Accuracy for the standard change-detection task. (B) Accuracy for the updating task. NUF, nonupdated feature; UF, updated feature; VSTM, visual short-term memory.

comparisons showed that, in valid trials, there was no significant difference in change-detection accuracy between updated $(75 \%)$ and nonupdated $(79 \%)$ objects in valid trials $[t(10)=1.05, p=.3, d=0.41]$. In NUF trials, there was no significant difference between updated objects $(64 \%)$ and nonupdated objects $(67 \%)[t(10)=1.06$, $p=.3, d=0.19]$. In UF trials, there was a significant advantage for updated objects $(91 \%)$ relative to nonupdated objects $(69 \%)[t(10)=6.97, p<.001, d=1.87]$. For updated objects, there was a significant difference between UF $(91 \%)$ and NUF $(64 \%)$ trials $[t(10)=7.06, p<.001$, $d=2.2]$ but not for nonupdated objects $[t(10)=1.19$, $p=.26, d=0.13]$. Similar to Experiment 1, these results demonstrate feature-selective updating.

With an additional analysis, we examined possible effects of task order on the updating task. For example, performing the memory task first may have influenced the 
participants to adopt an object-based strategy. The data were submitted to an order (first, second) $\times$ object (nonupdated, updated) $\times$ probe (valid, NUF, UF) mixed design ANOVA, which did not reveal a main effect of order $\left[F(1,9)=0.22, p=.65, \eta_{\mathrm{p}}^{2}=.024\right]$. More important, the three-way interaction was also not significant $[F(2,20)=$ $\left.0.88, p=.43, \eta_{\mathrm{p}}^{2}=.09\right]$, indicating no effect of task order on performance of the updating task. Together, these results provide evidence for object-based memory and feature-selective updating in the same participants.

\section{EXPERIMENT 3}

Another alternative explanation for the pattern of results observed in these experiments is that the participants strategically encoded information as separate features. A recent study showed that participants can selectively encode only task-relevant features of multifeature objects (Woodman \& Vogel, 2008). Furthermore, top-down factors have been well known to modulate the subjective parsing of information into objects (Chen \& Cave, 2006; Vecera \& Farah, 1994; Watson \& Kramer, 1999). It is possible that featureselective updating was a result of the participants' strategic encoding of information as separate features rather than as bound objects. Although object-based storage was found in Experiment 2, it is possible that the participants used different strategies for the two different tasks. In Experiment 3 , we included a condition in which the recurrence cue included both the updated and the nonupdated feature. In other words, the recurrence cue was a colored and oriented bar that appeared exactly like the updated object. For example, if the sample object was a blue vertical bar, the conjunction cue for a color update could be a red vertical bar, indicating an update to the color but no update to the orientation. We called these conjunction recurrence cues, in contrast to the feature recurrence cues that were used in the previous experiments. Note that for both types of cue, one feature of the object was updated (UF), whereas the other was not (NUF). Nonupdated features shown in the conjunction recurrence cues were shared with the sample object. To induce a bias toward object processing, the conjunction recurrence cues appeared on $70 \%$ of all trials and were randomly intermixed with feature recurrence cues. For conjunction recurrence cues, we predicted change-detection accuracy for the updated object to be superior across both the NUF and UF trials, because both features were present in the cue. Such high performance could result from updating in a truly object-based manner or from completely reencoding the entire object at the recurrence cue. We were critically interested in whether feature-selective updating would still be observed in the remaining $30 \%$ of trials that utilized the single-feature recurrence cues. Such results would further support the finding that feature-selective updating occurs despite the preponderance of object-based processing in the experiment.

\section{Method}

Participants. The participants were 11 Vanderbilt University undergraduate students ( 8 female), with a mean age of 19.09 years
$(S D=1.22)$. All of the participants were recruited via the procedures described in Experiment 2.

Apparatus and Stimuli. The apparatus and stimuli were identical to those in Experiment 1 except that, instead of appearing in three fixed locations, the objects were randomly positioned on 10 equidistant points of an imaginary circle with a diameter of $8.5^{\circ}$ visual angle.

Procedure. The procedure was identical to that in Experiment 1, except that, in $70 \%$ of the trials, the recurrence cue appeared as a colored and oriented bar. It appeared exactly like the updated object, including both updated and nonupdated features.

\section{Results and Discussion}

The data were scored as the proportion accurate and were submitted to a three-way ANOVA to examine the effects of recurrence type (conjunction, feature), object (updated, nonupdated), and probe (valid, NUF, UF). This analysis showed main effects of recurrence type $[F(1,10)=$ $\left.54.01, p<.001, \eta_{\mathrm{p}}^{2}=.84\right]$ and object $[F(1,10)=13.39$, $\left.p<.01, \eta_{\mathrm{p}}^{2}=.57\right]$ but not probe $[F(2,20)=0.8, p=$ $\left..46, \eta_{\mathrm{p}}^{2}=.07\right]$. There were significant two-way interactions for recurrence type and object $[F(1,10)=9.88, p<$ $\left..05, \eta_{\mathrm{p}}^{2}=.5\right]$, type and probe $[F(2,20)=5.74, p<.05$, $\left.\eta_{\mathrm{p}}^{2}=.36\right]$, and object and probe $[F(2,20)=10.32, p<$ $\left..001, \eta_{\mathrm{p}}^{2}=.5\right]$. More important, the three-way interaction was significant $\left[F(2,20)=7.75, p<.01, \eta_{\mathrm{p}}^{2}=.44\right]$. This suggested that differential behavior toward updated and nonupdated objects across different probe types were modulated by the type of recurrence cue involved in the trial (Figures 5A and 5B).

For a closer look at this result, separate $2 \times 3$ ANOVAs were conducted for each of the recurrence cue types to examine effects of object (updated, nonupdated) and probe (valid, NUF, UF). For trials involving the conjunction recurrence cues, the results showed a main effect only of object $\left[F(1,10)=38.38, p<.001, \eta_{\mathrm{p}}^{2}=.79\right]$. The main effect of probe $\left[F(2,20)=0.78, p=.47, \eta_{\mathrm{p}}^{2}=.07\right]$ and the interaction $\left[F(2,20)=0.44, p=.65, \eta_{\mathrm{p}}^{2}=.05\right]$ were not significant. Paired comparisons showed superior change-detection accuracy for updated $(94 \%)$ relative to nonupdated objects $(77 \%)[t(10)=6.19, p<.0001, d=$ $2.33]$. These results confirmed the prediction of superior memory for updated objects, without any difference in memory for the features of the updated objects. For trials with feature recurrence cues, the results did not show a main effect of object $\left[F(1,10)=0.6, p=.45, \eta_{\mathrm{p}}^{2}=.06\right]$ or probe $\left[F(2,20)=2.4, p=.11, \eta_{\mathrm{p}}^{2}=.19\right]$ but did show a significant object $\times$ probe interaction $[F(2,20)=12.46$, $\left.p<.001, \eta_{\mathrm{p}}^{2}=.55\right]$, supporting the feature-selective hypothesis. Paired comparisons showed no difference between updated and nonupdated objects in the valid trials $[t(10)=1.12, p=.28, d=0.43]$ or NUF trials $[t(10)=$ $0.99, p=.35, d=0.28]$. However, there was superior change detection for updated objects $(95 \%)$ relative to nonupdated objects $(72 \%)$ in the UF trials $[t(10)=4.7$, $p<.001, d=2.09]$. There was superior change-detection accuracy in UF trials $(94 \%)$ relative to NUF trials $(68 \%)$ for updated objects $[t(10)=3.65, p<.01, d=1.3]$ but not for nonupdated objects $[t(10)=0.35, p=.73, d=$ $0.12]$. These results confirm that updating was feature se- 


\section{A Experiment 3: Conjunction Recurrence Cues}

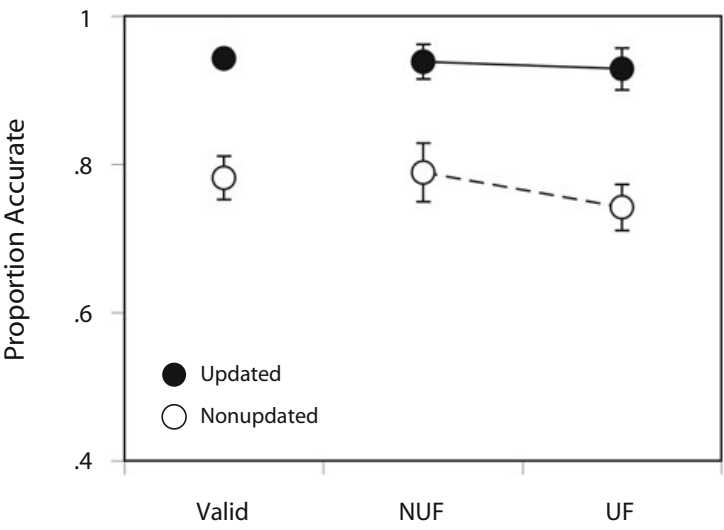

\section{B Experiment 3: Feature Recurrence Cues}

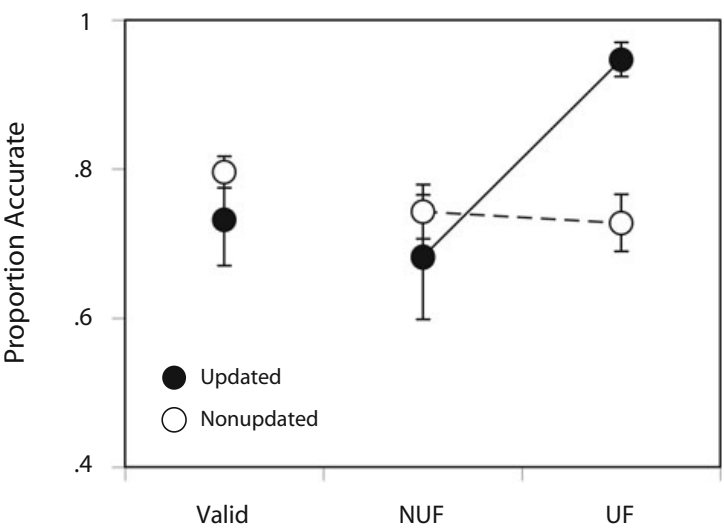

Figure 5. Results from Experiment 3. (A) Accuracy data for trials with conjunction recurrence cues. (B) Accuracy for trials with single-feature recurrence cues. NUF, nonupdated feature; UF, updated feature.

lective, just as in previous experiments. Together, these results suggest that, despite encouraging the encoding of information as whole objects by biasing the proportion of trials with conjunction recurrence cues, updating still proceeded in a feature-selective manner.

\section{EXPERIMENT 4}

Another alternative account of the feature-selective effect is that the participants may not have represented the updated appearance of the updated object. The participants may have merely represented the sample array and the recurrence cue but never integrated the two to form an updated representation. For example, if a trial consisted of updating a tilted blue bar into a vertical blue bar, as in Figure 1A, the participants may have only represented the tilted blue bar and the white vertical bar in memory and nothing more. This is in contrast to the task instructions, which emphasized integrating the new feature value into the memory of the object to create a representation of the updated object - in this example, a vertical blue bar. Although we do not doubt that the participants encoded and represented the objects in the sample array and the recurrence cue during the trial, the purpose of Experiment 4 was to demonstrate that they were also representing the updated appearance of the updated object.

To find evidence for a representation of the updated version of the updated object, we devised a task with the assumption that information in memory guides visual selection and that stronger representations have more influence on visual selection than do weaker representations. Participants readily attend to visual information that appears while they are currently representing that information in working memory (Downing, 2000; Huang \& Pashler, 2007; Soto, Heinke, Humphreys, \& Blanco, 2005), and this allocation of attention is automatic and can facilitate responses in an easy visual task (Olivers, Meijer, \& Theeuwes, 2006; Soto, Humphreys, \& Heinke, 2006; see Soto, Hodsoll, Rotshtein, \& Humphreys, 2008, for review). In Experiment 4, the trials proceed just as they did in the standard task (Figure 6A), except that on half of the trials, the participants completed a different task. On these trials, a vertical line appeared with the probe. This cued the participants to abandon the memory task and, instead, to make a simple speeded response as to whether the probe appeared to the right or left of the line (Figure 6B). For our critical comparison, we added another type of invalid trial, which was the unaltered version of the updated object (Figure 6A). The unaltered probe was identical to the object in the sample array. Following our hypothesis that the updated appearance of the updated object was represented in memory, we predicted that performances on the spatial task should be equivalent or better when the probe was the updated object relative to when it was the unaltered object.

\section{Method}

Participants. The participants were 24 Vanderbilt University undergraduate students ( 11 female), with a mean age of 18.83 years $(S D=0.81)$. All of the participants were recruited via the procedures described in Experiment 2.

Apparatus and Stimuli. The apparatus and stimuli were identical to those of Experiment 1, except for the following differences. Objects now appeared in three randomly selected locations for each trial. They were centered on 10 equidistant points of an imaginary circle that was $10^{\circ}$ visual angle in diameter. The objects in a sample were separated by at least one point on this circle and never appeared on the top or bottom location. This slight alteration was incorporated to accommodate the spatial task. The vertical line used in the spatial task subtended $1.5^{\circ} \times 10^{\circ}$ visual angle. The horizontal position of the line was $2.5^{\circ}$ visual angle to the left or right of the probe.

Procedure. The procedure was identical to that of Experiment 1, except for the following changes. On invalid trials, the applied changes were feature values from the opposite side of feature space as the valid values, to increase discriminability. There were three types of invalid trials, including NUF and UF trials, which were previously described. In the third type - unaltered trials - if an updated object was probed, the probe was identical to the object as it appeared in the sample display, as if it had never been updated. Alternatively, if a nonupdated object was probed in this third type of trial, the probe appeared with the updated feature incorporated into its appearance. 


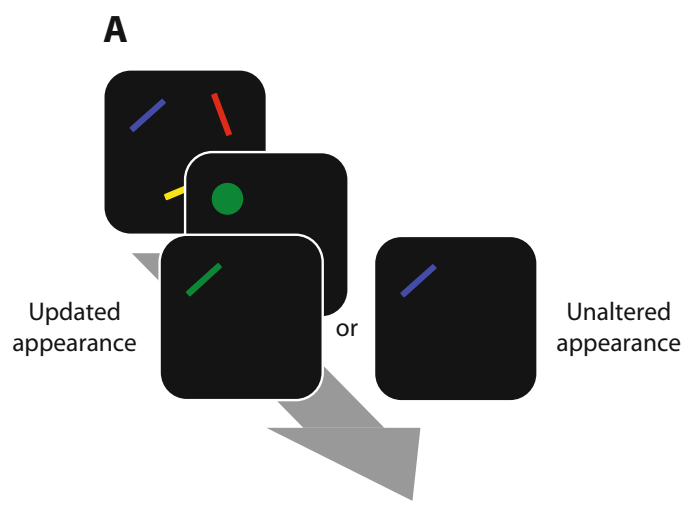

B

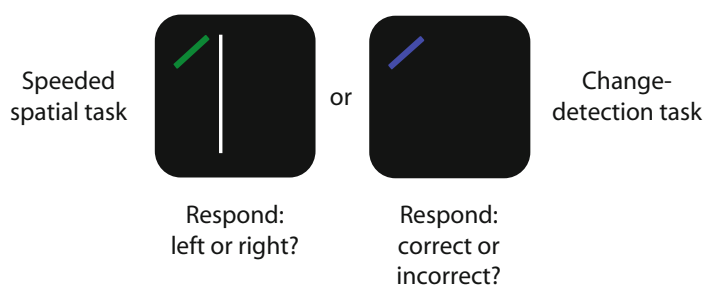

Figure 6. A schematic of the method of Experiment 4. (A) One trial over time, ending with the updated object in its updated appearance on the left (to which the participants would correctly respond correct), or with the unaltered appearance on the right (to which they would respond incorrect). (B) The two different tasks in which the participants might engage at the end of the trial. On the left, the appearance of a vertical line, randomly appearing on the left or right of the probed object, cued the participants to perform a speeded spatial judgment task. On the right, they engaged in the change-detection task (see the text for details).

The participants were cued to engage in either the changedetection task or a speeded spatial task on an equal number of trials (288 trials per task, 576 trials in total). As in the previous experiments, the probe appeared in the same location as its corresponding object in the sample. In the change-detection task, the probe appeared alone (Figure 5B), and the participants reported whether the probe appeared correctly or incorrectly by pressing keys marked "correct" or "incorrect" with their right hand. The participants were told that the change-detection task was unspeeded and to respond as accurately as possible. For the speeded spatial task, a thin, vertical white line appeared close to left or right of the probe's location (Figure 6B). In other words, wherever the probe appeared on the screen, the line appeared close to the probe's location and was equally likely to appear to the left or right of that location. The participants reported whether the probe appeared to the right or left of the line by pressing keys marked "left" or "right" with their left hand. They were told to respond as quickly as possible without sacrificing accuracy. They were instructed to keep their responding fingers over the keys during the experiment.

\section{Results and Discussion}

Responses were scored as the proportion accurate for both the change-detection and spatial tasks (Figures 7A and $7 \mathrm{~B})$.

The change-detection task. The accuracy data for the change-detection task were submitted to a $2 \times 4$ ANOVA to examine the effects of object (updated, nonupdated) and probe (valid, NUF, UF, unaltered). The results showed main effects of object $\left[F(1,23)=26.5, p<.001, \eta_{\mathrm{p}}^{2}=.54\right]$ and probe $\left[F(3,69)=2.9, p<.05, \eta_{\mathrm{p}}^{2}=.11\right]$. The object $\times$ probe interaction was significant $\left[F(3,69)=15.8, p<.001, \eta_{\mathrm{p}}^{2}=\right.$ $.41]$, once again supporting the feature-selective account of memory updating. There was no difference between updated and nonupdated objects in the valid trials $[t(23)=1.36, p=$ $.18, d=0.24]$ or NUF trials $[t(23)=1.12, p=.27, d=$ $0.23]$. For UF trials, change-detection accuracy was superior for updated objects $(80.9 \%)$ than for nonupdated objects $(56 \%)[t(23)=7.84, p<.001, d=1.71]$. For updated objects, accuracy for UF trials $(80.9 \%)$ was superior to that for NUF trials $(57.3 \%)[t(23)=5.38, p<.001, d=1.11]$. For nonupdated objects, there was no significant difference between NUF trials and UF trials (according to the Bonferronicorrected alpha level of .007) $[t(23)=2.5, p=.02, d=$ $0.37]$. These results replicate the previous three experiments supporting feature-selective updating. Finally, analysis of the change-detection task also involved the unaltered probe trials. Since the unaltered probes entailed a change to the updated feature, we predicted no difference between these and UF trials. This was confirmed by paired comparisons showing no significant differences between accuracies for unaltered probe trials and those for UF trials for both updated objects $[t(23)=1.87, p=.07, d=0.38]$ and nonupdated objects $[t(23)=1.42, p=.17, d=0.2]$.

The spatial task. For the spatial task, the data were scored as error rates and reaction times. For each participant, the reaction times for only correct trials were analyzed, and the remaining data were trimmed to exclude reaction times beyond $2.5 \mathrm{SDs}$ of the individual participant's grand mean. This resulted in a mean of $3.64 \%$ of trials $(S D=6.3 \%)$ being excluded. Since error rates and reaction time data were assumed to be measures of spatial task processing, they were jointly submitted to a $2 \times 4$ MANOVA to examine the effects of object (updated, nonupdated) and probe (valid, NUF, UF, unaltered). There was no main effect of object [Pillai's trace $=.019, F(2,183)=$ $1.8, p=.168$ ] or probe [Pillai's trace $=.009, F(2,183)=$ $0.269, p=.951]$ and the interaction was not significant [Pillai's trace $=.013, F(3,184)=0.386, p=.888$ ]. These null results may have been observed, because in the joint analysis, the overall variance of the reaction time data (total $S D=147.43$ ) was much greater than that of the error rate data (total $S D=.089$ ), obscuring important effects. Additionally, the assumption of homoscedasticity was violated (Box's $M=47.3, p=.001$ ), suggesting that these data may not be appropriate for the MANOVA (although Pillai's trace should be robust to this violation). We then submitted accuracy and reaction time data to separate univariate tests to determine whether effects were evident in each. Accuracy showed main effects of object $\left[F(1,23)=6.49, p<.05, \eta_{\mathrm{p}}^{2}=.22\right]$ and probe $[F(3,69)=$ $\left.3.06, p<.05, \eta_{\mathrm{p}}^{2}=.12\right]$, and the object $\times$ probe interaction was significant $\left[F(3,69)=3.31, p<.05, \eta_{\mathrm{p}}^{2}=.16\right]$. The reaction time data showed a main effect of object $\left[F(1,23)=10.98, p<.01, \eta_{\mathrm{p}}^{2}=.32\right]$ but no main effect of probe $\left[F(3,69)=0.87, p=.46, \eta_{\mathrm{p}}^{2}=.04\right]$. Although the 


\section{A Experiment 4: Change-Detection Task}

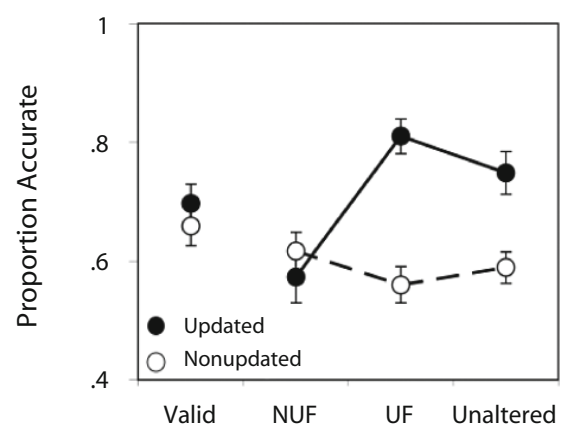

B Experiment 4: Speeded Spatial Task

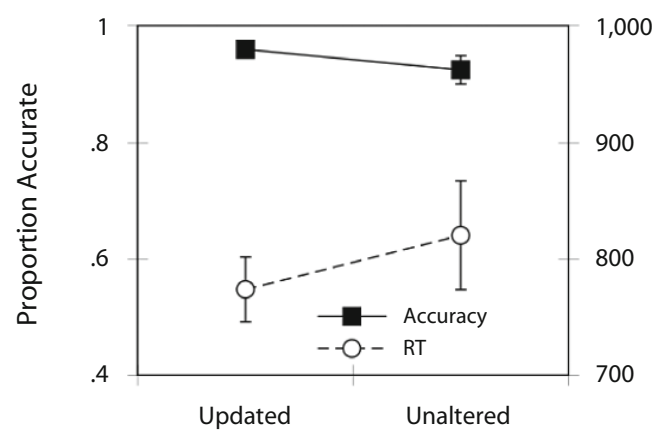

Figure 7. Results from Experiment 4. (A) Accuracy data for the change-detection task. (B) The critical contrasts for the speeded spatial judgment. NUF, nonupdated feature; UF, updated feature; RT, reaction time.

object $\times$ probe interaction was not significant $[F(3,69)=$ $\left.2.58, p=.06, \eta_{\mathrm{p}}^{2}=.1\right]$, it approached significance, suggesting that the variance in the reaction time data may have obscured underlying effects.

The primary contrast of interest was between the updated and unaltered probes of the updated object (see Figure 7B). We hypothesized that if the participants represented the updated appearance of the updated object, their spatial task performance to that updated probe should be equal to, or perhaps better than that to the unaltered probe. Accuracy data showed superior performances for updated probes $(96 \%)$ than for unaltered probes $(92 \%)[t(23)=2.17, p<.05, d=0.36]$. Reaction times showed no difference between updated and unaltered probes $[t(23)=1.7, p=.1, d=0.25]$. However, the trend in the means was for faster reaction times with the updated form $(\bar{X}=774 \mathrm{msec}, S E=28)$ than with the unaltered form $(\bar{X}=820 \mathrm{msec}, S E=47)$, suggesting that reaction time behavior reflected the same effect found in accuracy and not a speed-accuracy tradeoff. These results confirmed our prediction that task performances for the updated object probe were at least as good, if not better, than performances for the unaltered probe, suggesting that the updated appearance of the ob- ject was represented at least as well as the unaltered appearance. Responses to the spatial task were consistent with the idea that the participants were integrating the information about the recurrence cue with the sample array to create a memory representation of the updated object. It is also possible, however, that memory of the unaltered object was suppressed by the appearance of the recurrence cue, leading to worse performance on the speeded localization task. As such, feature information about the recurrence cue may not have been integrated with the sample information at all. We return to this possibility in the General Discussion section.

\section{EXPERIMENT 5}

The results of the previous experiments have consistently shown an advantage in accuracy for UF trials over NUF trials, specifically for updated objects and not for nonupdated objects. We have interpreted this effect as the facilitation of memory for one feature of the updated object, which does not spread to its remaining features. In other words, updating a single feature of an object does not refresh the memory of the entire object. An alternative account is that the observed increase in sensitivity to changes in the feature value associated with the recurrence cue was due to the cue's recent appearance. In other words, perhaps the participants had the best memory for the updated feature, because the recency of the recurrence cue facilitated memory for this feature in general, not specifically for the updated object. This recency account can be considered a form of the feature-based account described in the introduction, which proposed that updating facilitates detection for all values of the updated feature dimension (e.g., orientation). The feature-based account is modified here to propose that the facilitation occurs only for the specific feature in the recurrence cue (e.g., vertical). The difference between this recency account and the feature-selective account is that the latter predicts increased accuracy specifically for the updated object, whereas the former predicts the advantage for any object with the same feature as the recurrence cue. Such an account accurately describes the results of the previous experiments, because the updated object was always the only object with the same feature as the recurrence cue.

We examined this account by incorporating a nonupdated object with a feature common to that of the recurrence cue. For example, consider an example trial in which the sample array contained a red vertical bar, a blue horizontal bar, and a yellow tilted bar, followed by a recurrence cue indicating that the orientation of the yellow bar should be updated to horizontal. The blue horizontal bar is a nonupdated object that shares a feature with the recurrence cue (called nonupdated shared object), whereas the red vertical bar is a nonupdated object that does not share features with the other objects (a nonupdated unshared object). In this situation, the recency account predicts a bias to remember the horizontal orientation, regardless of whether it applied to the updated object or to the nonupdated object with the shared feature. The feature-selective account 
predicts increased accuracy at remembering the horizontal orientation only as it applies to the updated object.

\section{Method}

Participants. The participants were 11 Vanderbilt University undergraduate students ( 9 female), with a mean age of 18.36 years $(S D=$ 0.81 ), recruited via the procedures described in Experiment 2.

Apparatus and Stimuli. The apparatus and stimuli were identical to those of Experiment 1, except for the following changes. To better match the discriminability of the colors, orientations were now comprised of 12 angles increasing from zero at $15^{\circ}$ increments. Only second-neighbor orientations appeared in a given trial. For both color and orientation, updates and invalid feature values were drawn from the same pool as the sample feature values but were novel to the trial.

Procedure. The procedure was identical to that of Experiment 1, except for the following changes. In half of the trials, one of the nonupdated objects shared a feature with the recurrence cue. For example, if the recurrence cue was a horizontal line, one of the nonupdated objects had a horizontal orientation. This condition was called the shared condition. The other nonupdated object did not share any features with the recurrence cue or any other object, so the shared trials contained both a shared and an unshared nonupdated object. In the other half of the trials (the unshared trials), neither nonupdated object shared features with the recurrence cue.

\section{Results and Discussion}

The data were scored as the proportion accurate and were separated according to whether the recurrence cue shared a feature with a nonupdated object or not (Figures $8 \mathrm{~A}$ and $8 \mathrm{~B}$ ). Note that unshared trials were essentially a replication of Experiment 1 . The unshared trials were submitted to a $2 \times 3$ ANOVA to examine the effects of object (updated, nonupdated) and probe (valid, NUF, UF). There were main effects of object $[F(1,10)=7.53$, $\left.p<.05, \eta_{\mathrm{p}}^{2}=.43\right]$ and probe $[F(2,20)=7.6, p<.01$, $\left.\eta_{\mathrm{p}}^{2}=.43\right]$ and a significant interaction $[F(2,20)=4.91$, $\left.p<.05, \eta_{\mathrm{p}}^{2}=.33\right]$. Paired comparisons did not show a significant difference between updated and nonupdated objects in valid trials $[t(10)=2.25, p=.047, d=0.95]$ (with an adjusted $\alpha=.01$ ) or in NUF trials $[t(10)=1.01$, $p=.33, d=0.58]$ but there was a significant difference in UF trials $[t(10)=5.2, p<.001, d=1.73]$. Changedetection accuracies for UF trials were superior to those for NUF trials for updated objects $[t(10)=4.39, p<.01$, $d=1.71]$ but not for nonupdated objects $[t(10)=0.45$, $p=.66, d=0.15]$. This pattern of results replicates those of our previous experiments.

The shared trials were submitted to a $3 \times 3$ ANOVA to examine the effects of object (updated, nonupdated shared, nonupdated unshared) and probe (valid, NUF, UF). Remember that every trial had two nonupdated objects, so, even for shared trials, there was an unshared object. The results showed a main effect of object $[F(2,20)=$ $\left.7.34, p<.01, \eta_{\mathrm{p}}^{2}=.42\right]$ but not of probe $[F(2,20)=2.9$, $\left.p=.07, \eta_{\mathrm{p}}^{2}=.22\right]$. There was a significant interaction $\left[F(4,40)=4.98, p<.001, \eta_{\mathrm{p}}^{2}=.33\right]$. Paired comparisons showed change-detection accuracies to be superior in UF trials than in NUF trials for updated objects $[t(10)=3.93$, $p<.004, d=1.8]$ but not for nonupdated shared $[t(10)=$ 1.6, $p=.14, d=0.5]$ or nonupdated unshared $[t(10)=$ $0.5, p=.11, d=0.16]$ objects. In valid trials, there were no significant differences between accuracies for updated and those for nonupdated shared objects $[t(10)=1.01$, $p=.33, d=0.44]$ or between those for updated and those for nonupdated unshared objects $[t(10)=2.09, p=.06$, $d=0.84]$. In NUF trials, there were no significant differences between accuracies for updated and those for nonupdated shared objects $[t(10)=1.17, p=.27, d=0.55]$ or between those for updated and those for nonupdated unshared objects $[t(10)=0.99, p=.34, d=0.44]$. In UF trials, there were significant differences between accuracies for updated and those for nonupdated shared objects $[t(10)=6.28, p<.001, d=2.35]$ and between those for updated and those for nonupdated unshared objects $[t(10)=10.43, p<.001, d=3.42]$. There was no difference between the accuracies of the two kinds of nonupdated objects (shared and unshared) $[t(10)=0.95, p=$ $.36, d=0.31]$.

These results revealed higher accuracies for UF trials than for NUF trials for updated objects but not for nonupdated objects that shared a feature with the recurrence cue. This result is predicted by the feature-selective hypothesis but not by the recency account. Furthermore, there was no difference between these conditions for nonupdated objects that did not share features with the recurrence cue, which resembles results from our previous experiments. Thus, the observed pattern of results cannot be attributed to a general tendency to remember the specific feature in the recurrence cue. Instead, the results must reflect better performances for the updated feature in the updated object than for any other feature, which we are calling featureselective updating.

\section{EXPERIMENT 6}

The results of Experiment 5 ruled out a recency account, which proposed that the results reflected increased sensitivity to the specific feature value of the recurrence cue, regardless of its corresponding object. To further rule out this account, we conducted another experiment, in which the recurrence cue did not resemble any possible target features. Instead, the recurrence cues were letters associated with the features. For example, participants were shown the letter " $R$ " to cue the updating of color to red. This methodology encourages verbal encoding strategies, so we removed the articulatory suppression task. In this way, in Experiment 6, we also examined whether featureselective updating can occur for working memory more generally, without isolating VSTM specifically.

\section{Method}

Participants. The participants were 13 Vanderbilt University undergraduate students (10 female), with a mean age of 18.69 years $(S D=0.75)$. All of the participants were recruited via the procedures described in Experiment 2.

Apparatus and Stimuli. The apparatus and stimuli were identical to those in Experiment 1, except for the following changes. Feature values were now restricted to four colors and orientations. The colors were not controlled for discriminability and, instead, were canonical primary colors constructed with the following RGB values: red $(255,0,0)$, blue $(0,0,255)$, green $(0,255,0)$, yellow $(255,255,0)$. The orientations were $0^{\circ}, 45^{\circ}, 90^{\circ}$, or $135^{\circ}$ (where $0^{\circ}$ is vertical).

Procedure. The procedure consisted of the learning phase and the testing phase. In the learning phase, the participants learned the 
A

Experiment 5: Unshared Features

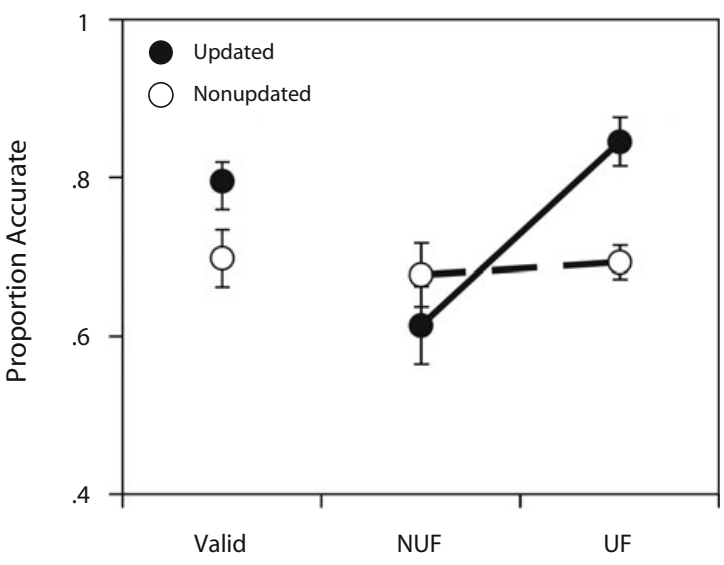

B

Experiment 5: Shared Features

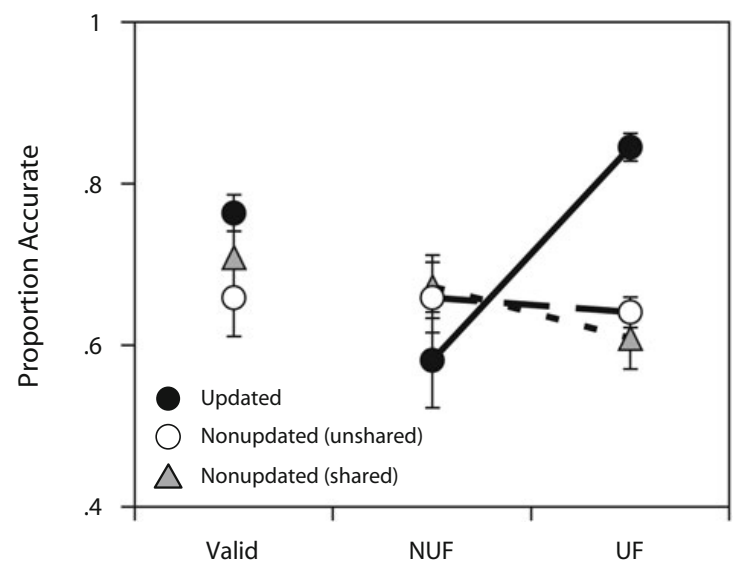

Figure 8. Results from Experiment 5. (A) Accuracy data for the trials in which nonupdated objects did not share any features with the recurrence cue. (B) Accuracy data for trials with one nonupdated object that shared a feature with the recurrence cue and with one that did not share features. NUF, nonupdated feature; UF, updated feature.

association between the letter cues and the object features. First, the participants studied a table displaying the letters to be used as the updating cues and the visual features for which they stood. The letters used were R (red), G (green), B (blue), Y (yellow), H (horizontal), V (vertical), E (eastward), and W (westward). The last two letters stood for orientations at $45^{\circ}$ and $135^{\circ}$, respectively. For these orientation values, the participants were given a mnemonic to help them remember the cues, which was to imagine that the top of the bar was a compass pointing either to the east or to the west. Second, the participants engaged in a computerized learning task designed to strengthen the association between the letters and the visual features. Each trial in the task began with the presentation of a colored and oriented bar presented at the center of the screen for $200 \mathrm{msec}$, followed by blank 200 -msec ISI. This was followed by the central presentation of one of the eight letters. The participants' task was to report whether the visual feature symbolized by the letter had been present in the bar by pressing keys marked "yes" or "no." In half of the trials, the feature denoted by the letter was absent in the bar. There were 10 repetitions of each of the 16 conditions in each block. After each block, the experimenter informed the participants to which cues they had responded with less than $90 \%$ accuracy. The participants were tested with two blocks of the task, and additional blocks were added if they had not reached $90 \%$ accuracy on all cues by the second block (only 1 participant required an additional block to reach $90 \%$ accuracy). In the testing phase, the procedure was identical to that in Experiment 1, except that there was no verbal suppression task, and the recurrence cues were letters instead of bars or dots.

\section{Results and Discussion}

The results for the learning phase demonstrated that the participants learned the symbolic letter cues easily. Average accuracy across all cues was $97.84 \%$ and the average reaction time was $834 \mathrm{msec}$. The mean accuracies and reaction times for each letter cue are reported in Table 1.

From the testing phase, the change-detection accuracy data were submitted to a $2 \times 3$ ANOVA to examine the effects of object (nonupdated, updated) and probe (valid, NUF, UF). The results showed a main effect of object $\left[F(1,12)=16.9, p<.01, \eta_{\mathrm{p}}^{2}=.58\right]$ but no effect of probe $\left[F(2,24)=3.03, p=.07, \eta_{\mathrm{p}}^{2}=.20\right]$. A significant object $\times$ probe interaction $\left[F(2,24)=16.5, p<.001, \eta_{\mathrm{p}}^{2}=.58\right]$ once again showed the feature-selective effect of updating (Figure 9). Paired comparisons showed no difference between accuracies for updated and nonupdated objects in valid trials $[t(12)=0.08, p=.94, d=0.025]$ and in NUF trials $[t(12)=1.64, p=.12, d=0.58]$. However, in UF trials, change-detection accuracy for updated objects $(95 \%)$ was superior to that for nonupdated objects $(70 \%)$ $[t(12)=7.5, p<.001, d=3.13]$. Change-detection accuracies for updated objects were superior in UF trials than in NUF trials $[t(12)=3.99, p<.01, d=1.26]$, whereas there was no difference between accuracies in UF trials and those in NUF trails for nonupdated objects $[t(12)=$ $1.28, p=.22, d=0.2]$. The results of this experiment replicated the feature-selective updating effect while further ruling out the recency account. In addition, this result suggests that feature-selective updating may generalize to other aspects of working memory, in addition to purely visual information.

\section{GENERAL DISCUSSION}

In this study, we examined whether updating VSTM was an object-based process - namely, whether updating a single feature of an object in memory would lead to refreshing all of the features of that object. The main finding was that change detection for an updated feature was superior to that for the nonupdated feature of the updated object. In fact, memory for the nonupdated feature of the updated object was no better than were features of the other objects. We take these findings to indicate that, under these conditions, updating is not an object-based process but, rather, was solely restricted to the updated feature. We called this effect feature-selective updating (Experiment 1). We further showed that this effect was not due to failure to store information as objects (Experiment 2) and that it was still observed even when object-based 
processing was strongly encouraged (Experiment 3 ). We presented evidence to suggest that feature-selective updating was unlikely to be due to a poor representation of the updated object (Experiment 4). The recency of the updated feature did not affect just any object in memory but was specific to the updated object (Experiments 5), and the feature-selective effect was even observed with verbal rather than visual cues (Experiment 6). In summary, we consistently observed results suggesting that the facilitative effects of updating were restricted to the memory of the updated feature and did not lead to reprocessing of the whole updated object. We interpreted these results to indicate that updating VSTM is feature selective and not object based. Although we conducted several experiments to rule out alternative accounts to feature-selective updating, the present results are consistent with at least two possible accounts. Feature-selective updating might indicate that representations in VSTM are not objects but features. Alternatively, updating and storage mechanisms of VSTM may have different bases. We will discuss both of these possibilities in turn.

\section{Are There Object-Based \\ Representations in VSTM?}

The present results showing feature-selective updating call into question the type of representations used in VSTM and may suggest that information in VSTM is represented as features rather than as objects. The theory that object-based representations are the basis for the storage of information in VSTM has been intensely debated. The groundbreaking discovery of object-based representations, by Luck and Vogel (1997), demonstrated that two features of an object can be stored in VSTM with the same memory load as a single feature. This result could be accounted for by strong associations between the memory for the different features that make up an object, such that remembering one feature automatically gives rise to the other at no cost. More recent results show that costs related to the number of features are particularly detrimental to this strong version of the theory. For example, Olson and Jiang (2002) showed that storing color and orientation features as unified objects provided a modest benefit over storing the exact same features as separate items. Storing

Table 1

Mean Proportions Accurate and Reaction Times (RTs, in Milliseconds) for Each Cue of the Learning Task in Experiment 5

\begin{tabular}{cccccc}
\hline & \multicolumn{2}{c}{ Accuracy } & & \multicolumn{2}{c}{ RT } \\
\cline { 2 - 3 } \cline { 5 - 5 } Cue & $M$ & SEM & & $M$ & SEM \\
\hline R & .99 & .007 & & 790.62 & 94.08 \\
B & .98 & .012 & & 735.02 & 52.53 \\
G & .97 & .009 & & 748.41 & 58.18 \\
Y & .99 & .005 & & 777.36 & 68.77 \\
H & .98 & .009 & & 898.14 & 98.66 \\
W & .97 & .012 & & 915.98 & 97.44 \\
V & .99 & .005 & & 810.08 & 80.13 \\
E & .95 & .017 & & $1,003.10$ & 125.24 \\
\hline
\end{tabular}

Note-R, red; B, blue; G, green; Y, yellow; H, horizontal; W, westward; $\mathrm{V}$, vertical; E, eastward.

\section{Experiment 6: Letter Recurrences}

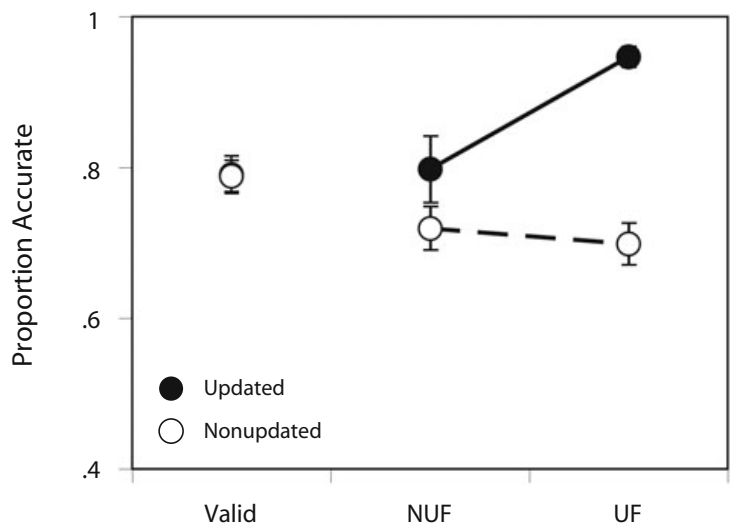

Figure 9. Results from Experiment 6. NUF, nonupdated feature; UF, updated feature.

the information as objects did not cut the cost of storage in half, suggesting that the number of features also exerted a cost for storage. Similarly, Xu (2002) showed storage costs related to the multiple parts of objects. Alvarez and Cavanagh (2004) showed that VSTM storage capacity decreased as stimulus complexity increased (but see Eng, Chen, \& Jiang, 2005). If objects in memory are the associations formed between its component features, such results indicate that the associations are not perfect, because increasing the number of features also increases the memory load. However, in all of this research on memory storage, at least some benefit of object-based organization of features was found, even if it was imperfect. Given the wealth of evidence supporting an object-based benefit to storage of information in VSTM, it is unlikely that our findings of feature-selective updating indicate that objectbased storage does not exist. It is more likely that our findings reflect the possibility that storage and updating processes in VSTM do not necessarily share a common basis of representation. In other words, VSTM storage may be object-based, whereas VSTM updating could be featurebased. In the next section, we discuss possible ways that this could be implemented.

\section{Different Bases for Updating and Storage}

It is possible that whereas working memory storage is object-based, updating is instead feature-based. One way this could be possible is if the selection of a single feature of an object for updating breaks the associations between features formed at encoding and maintained in storage. Previous research has shown that features of perceived objects can be selectively encoded into VSTM according to the task instructions (Woodman \& Vogel, 2008), and it remains plausible that similar feature-selective behavior can occur when the objects are already stored in memory. In addition to the present study, others have suggested that updating to be mediated by distinct, feature-specific systems (Mohr, Goebel, \& Linden, 2006; Mohr \& Linden, 2005). Mohr and 
Linden examined updating of visual memory by presenting participants with two objects, each with a unique color and orientation, and instructing them to imagine a mix of the colors or the average of the two orientations or both. Their results showed no dual-task cost between tasks requiring both color and orientation updating relative to when they only performed one updating task, but there was a dual-task cost when two orientation-updating tasks were performed. Furthermore, they showed that updating depended on central executive resources, whereas maintenance did not. Together, these results suggest that updating of visual memory is mediated by independent, feature-specific systems and may engage additional executive functions. These executive functions may allow the updating process to ignore or break associations between features formed at encoding and maintained in memory.

A useful way to speculate further how updating could break object representations is to consider known theories of object-based processing. Object file theory (Kahneman, Treisman, \& Gibbs, 1992) proposes that updating an object consists of three components: (1) correspondence, in which a perceived object is either a new or previously viewed object; (2) reviewing, in which, once correspondence is established, older information regarding the perceived object is retrieved; and (3) impletion, where the old and new information are integrated to generate the percept of one object changing, rather than two different objects. In our paradigm, we assumed that object correspondence between the sample object and the recurrence cue could easily be established by a common location. That all participants were able to understand and accomplish the memory updating task at greater than chance levels supports this assumption. The second stagereviewing - may have been impaired. Notice that this is another way of saying that the feature information in the recurrence cue was not successfully integrated with the information from the sample array. It is possible that the nature of the recurrence cue interfered with appropriate reviewing of all of the features of the object in memory because the recurrence cue was a surface feature, such as color or orientation. In classic object file studies, the reviewing process has been typically shown to retrieve a single instance of an object, whether it is a letter (Kahneman et al., 1992), an abstract identity (Gordon \& Irwin, 2000), or a single face (Mitroff, Scholl, \& Noles, 2007). The information about the object was, in this previous work, to be integrated across multiple spatial locations. In the present study, however, the task required integration of multiple instances of feature information (color or orientation) to be integrated within a single spatial location. Perhaps reviewing previous states of an object is best guided by integrating across previous locations but not across surface features, such as color and orientation. Or, in other words, the memory for nonupdated surface features is not refreshed. This was reflected in our results, which showed no impairment in change detection for the nonupdated feature of the updated object relative to that of the nonupdated objects.

Although the results of this study showed that updating one feature of an object did not refresh all of its other fea- tures, other research has shown that refreshing of nonupdated information can occur. Kessler and Meiran (2006) had participants remember two digits, each associated with a shape, and update their memory of each digit by periodically performing arithmetic operations (e.g., add 3). If an arithmetic instruction appeared in a shape, the participants updated their memory of the digit associated with that shape. Reaction time costs of updating a digit were larger in blocks of trials in which both shapes appeared relative to blocks in which only one shape ever appeared. Kessler and Meiran interpreted their results to indicate that updating included refreshing more of the contents of memory than just the information updated. Similarly, Bao et al. (2007) demonstrated that the reaction time costs of switching between two concurrent updating tasks were attenuated when the tasks involved the same object. The participants updated the location of an object following arrow instructions while doing arithmetic operations on a memorized digit. When the digit was used both for the location and for the arithmetic tasks, performance was improved, suggesting that updating a single object is easier than updating separate object representations in memory. Although the results of the present study suggest that updating could break associations between features of an object, it is possible that the associations could be sustained or reformed depending on the task demands. Kessler and Meiran proposed that updating disrupts the stability of working memory and that it would need to undergo further processing to become stable again. Updating could disrupt memory stability, because it demands a change to working memory contents, such that working memory would benefit from reconsolidation after updating. One difference between the present study and these others (Bao et al., 2007; Kessler \& Meiran, 2006), is that the procedure in these previous studies allowed participants as much time as they required to update their memories, whereas in the present study, we kept this time limited. Although other attributes of the procedure may provide the necessary conditions for memory reconsolidation to produce object-based updating, such factors are currently unknown, opening an avenue for potential research. The present results indicate that updating is not always objectbased and instead can be feature-selective.

\section{What Is the Mechanism for Updating?}

One mechanism arguably involved in memory updating is attentional selection. Oberauer (2002) proposed a concentric model of working memory in which information existed in three states: (1) an activated portion of long-term memory that represented the passive storage of information; (2) a smaller region of direct access, in which information was readily accessible for retrieval; and (3) the focus of attention, which holds just the chunk of information that is immediately available for processing. According to the concentric model, updating memory includes initially directing the focus of attention to the part of memory to be updated. There is a wealth of evidence showing that attention selects entire objects for processing (Duncan, 1984; Egly, Driver, \& Rafal, 1994; for a review, see Scholl, 2001). For example, Duncan (1984) showed that the cost of dividing attention 
between two features, such as texture and orientation, was alleviated when the features appeared on the same object, as opposed to on two different objects. One might expect, then, that attention allocated to memory representations, for the purpose of updating, would also be object based. However, some evidence suggests that part of object-based benefits in attentional allocation is driven by perceptual and stimulusrelated factors (Avrahami, 1999; Matsukura \& Vecera, 2006; Scholl, Pylyshyn, \& Feldman, 2001). Whether object-based attention or feature-based attention is used to select relevant parts of memory for updating is an empirical question. Our results support the idea that updating memory does not necessarily involve object-based attention but may be performed with attention to specific features.

\section{Conclusion}

This study showed that updating one feature of an object in VSTM did not lead to the automatic refreshing of the memory of its nonupdated features. In other words, updating objects in memory does not always lead to reprocessing of all of its attributes. It remains open for further research to determine how the mechanisms involved in updating can be described, given this observation that updating visual information is feature selective.

\section{AUTHOR NOTE}

Correspondence concerning this article should be addressed to P. C. Ko, Department of Psychology, Vanderbilt University, 11121 st Avenue South, Nashville, TN 37203 (e-mail: p.ko@vanderbilt.edu).

\section{REFERENCES}

Alvarez, G. A., \& Cavanagh, P. (2004). The capacity of visual shortterm memory is set both by visual information load and by number of objects. Psychological Science, 15, 106-111.

Avrahami, J. (1999). Objects of attention, objects of perception. Perception \& Psychophysics, 61, 1604-1612.

BAO, M., LI, Z.-H., \& ZHANG, D.-R. (2007). Binding facilitates attention switching within working memory. Journal of Experimental Psychology: Learning, Memory, \& Cognition, 33, 959-969.

Brainerd, D. H. (1997). The psychophysics toolbox. Spatial Vision, 10, 433-436.

Chen, Z., \& Cave, K. R. (2006). Reinstating object-based attention under positional certainty: The importance of subjective parsing. Perception \& Psychophysics, 68, 992-1003.

CIE (1986). Colorimetry (2nd ed., Publication 15.2 [E-1.3.1]). Vienna: Central Bureau of the CIE.

Cowan, N. (2001). The magical number 4 in short-term memory: A reconsideration of mental storage capacity. Behavioral \& Brain Sciences, 24, 87-114.

DownING, P. E. (2000). Interactions between visual working memory and selective attention. Psychological Science, 11, 467-473.

DunCAN, J. (1984). Selective attention and the organization of visual information. Journal of Experimental Psychology: General, 113, 501-517.

Egly, R., Driver, J., \& Rafal, R. D. (1994). Shifting visual attention between objects and locations: Evidence from normal and parietal lesion subjects. Journal of Experimental Psychology: General, 123, 161-177.

ENG, H. Y., CHEN, D., \& JIANG, Y. (2005). Visual working memory for simple and complex visual stimuli. Psychonomic Bulletin \& Review, 12, 1127-1133.

Friedman, N. P., Miyake, A., Corley, R. P., Young, S. E., DeFries, J. C., \& HewitT, J. K. (2005). Not all executive functions are related to intelligence. Psychological Science, 17, 172-179.

Garavan, H. (1998). Serial attention within working memory. Memory \& Cognition, 26, 263-276.

Gordon, R. D., \& IRWIN, D. E. (2000). The role of physical and concep- tual properties in preserving object continuity. Journal of Experimental Psychology: Learning, Memory, \& Cognition, 26, 136-150.

Griffin, I. C., \& Nobre, A. C. (2003). Orienting attention to locations in internal representations. Journal of Cognitive Neuroscience, 15, 1176-1194.

HuAng, L., \& PAShler, H. (2007). A boolean map theory of visual attention. Psychological Review, 114, 599-631.

Kahneman, D., Treisman, A., \& Gibbs, B. J. (1992). The reviewing of object files: Object-specific integration of information. Cognitive Psychology, 24, 174-219.

Kessler, Y., \& MeIRAN, N. (2006). All updateable objects in working memory are updated whenever any of them are modified: Evidence from the memory updating paradigm. Journal of Experimental Psychology: Learning, Memory, \& Cognition, 32, 570-585.

Landman, R., Spekreisse, H., \& Lamme, V. A. F. (2003). Large capacity storage of integrated objects before change blindness. Vision Research, 43, 149-164.

Luck, S. J., \& Vogel, E. K. (1997). The capacity of visual working memory for features and conjunctions. Nature, 390, 279-281.

MAKOVSKI, T., \& JIANG, Y. V. (2007). Distributing versus focusing attention in visual short-term memory. Psychonomic Bulletin \& Review, 14, 1072-1978.

Makovski, T., Sussman, R., \& Jiang, Y. V. (2008). Orienting attention in visual working memory reduces interference from memory probes. Journal of Experimental Psychology: Learning, Memory, \& Cognition, 34, 369-380.

Matsukura, M., \& Vecera, S. (2006). The return of object-based attention: Selection of multiple-region objects. Perception \& Psychophysics, 68, 1163-1175.

Mitroff, S. R., Scholl, B. J., \& Noles, N. S. (2007). Object files can be purely episodic. Perception, 36, 1730-1735.

Miyake, A., Friedman, N. P., Emerson, M. J., Witzki, A. H., \& HowERTER, A. (2000). The unity and diversity of executive functions and their contribution to complex "frontal lobe" tasks: A latent variable analysis. Cognitive Psychology, 41, 49-100.

Mohr, H. M., Goebel, R., \& Linden, D. E. J. (2006). Content- and task-specific dissociations of frontal activity during maintenance and manipulation in visual working memory. Journal of Neuroscience, 26, 4465-4471.

Mohr, H. M., \& Linden, D. E. J. (2005). Separation of the systems for color and spatial manipulation in working memory revealed by a dualtask procedure. Journal of Cognitive Neuroscience, 17, 335-366.

Morris, N., \& JonEs, D. M. (1990). Memory updating in working memory: The role of the central executive. British Journal of Psychology, 81, 111-121.

OBERAuER, K. (2002). Access to information in working memory: Exploring the focus of attention. Journal of Experimental Psychology: Learning, Memory, \& Cognition, 28, 411-421.

Olivers, C. N. L., Meijer, F., \& Theeuwes, J. (2006). Feature-based memory-driven attentional capture: Visual working memory content affects visual attention. Journal of Experimental Psychology: Human Perception \& Performance, 32, 1243-1265.

Olson, I. R., \& JiAng, Y. (2002). Is visual short-term memory object based? Rejection of the "strong-object" hypothesis. Perception \& Psychophysics, 64, 1055-1067.

Pelli, D. G. (1997). The VideoToolbox software for visual psychophysics: Transforming numbers into movies. Spatial Vision, 10, 437-442.

Schanda, J. (2007). Colorimetry: Understanding the CIE system. Hoboken, NJ: Wiley-Interscience.

SCHOLL, B. J. (2001). Objects and attention: The state of the art. Cognition, 80, 1-46.

Scholl, B. J., Pylyshyn, Z. W., \& Feldman, J. (2001). What is a visual object? Evidence from target merging in multiple object tracking. Cognition, 80, 159-177.

Soto, D., Heinke, D., Humphreys, G. W., \& Blanco, M. J. (2005). Early, involuntary top-down guidance of attention from working memory. Journal of Experimental Psychology: Human Perception \& Performance, 31, 248-261.

Soto, D., Hodsoll, J., Rotshtein, P., \& Humphreys, G. W. (2008). Automatic guidance of attention from working memory. Trends in Cognitive Sciences, 12, 342-348.

Soto, D., Humphreys, G. W., \& Heinke, D. (2006). Working memory can guide pop-out search. Vision Research, 46, 1010-1018. 
Vecera, S. P., \& Farah, M. J. (1994). Does visual attention select objects or locations? Journal of Experimental Psychology: General, 123, 146-160.

Vogel, E. K., Woodman, G. F., \& LucK, S. J. (2001). Storage of features, conjunctions, and objects in visual working memory. Journal of Experimental Psychology: Human Perception \& Performance, 27, 92-114.

Watson, S. E., \& Kramer, A. F. (1999). Object-based visual selective attention and perceptual organization. Perception \& Psychophysics, 61, 31-49.

Wheeler, M. E., \& Treisman, A. M. (2002). Binding in short-term visual memory. Journal of Experimental Psychology: General, 131, 48-64.

Woodman, G. F., \& Vogel, E. K. (2008). Selective storage and maintenance of an object's features in visual working memory. Psychonomic Bulletin \& Review, 15, 223-229.

XU, Y. (2002). Encoding color and shape from different parts of an ob- ject in visual short-term memory. Perception \& Psychophysics, 64, 1260-1280.

\section{NOTE}

1. Capacity estimates may not be theoretically appropriate for tasks using a whole array probe, as in the present experiment, because of the possibility of accumulated decision errors as set size increases. However, previous work has shown that decreasing performances due to set-size effects are not due to accumulated decision errors, but to capacity limitations (Vogel, Woodman, \& Luck, 2001). Similar results were obtained when the proportion accurate data was submitted to a stimulus $\times$ set size ANOVA, showing the same main effects and a marginal interaction.

(Manuscript received June 11, 2008;

revision accepted for publication February 26, 2009.) 